\title{
A Framework for Real-Time Resource Allocation in IP Multimedia Subsystem Network
}

\author{
Muhammad Naeem \\ Department of Computer Science, Mohammad Ali Jinnah University, Islamabad, Pakistan \\ naeems.naeem@gmail.com \\ Sheneela Naz and Saira Gillani \\ Centre of Research in Networks \& Telecom, Mohammad Ali Jinnah University Islamabad, Pakistan \\ shahneela.cs@gmail.com, sairagilani@yahoo.com
}

\begin{abstract}
IMS (IP Multimedia Subsystem) is designed to provide multiple-services and can be accessed from any access network. With the advent of new multimedia services; user requirement also changed and required delay tolerant services from the service providers. The new multimedia services demands the restrictions on network parameters and this can be achieved by analyzing the traffic flow from the core as well as access network capabilities. As access and core IMS network are independent of each other and does not exchange user SLA with each other. In this paper we proposed framework based on Session border controller (SBC) that proactively monitored the traffic statistics as well as interact with access network operator for getting user's SLA and reserve resources accordingly. We are also proposing alterations in SIP invite and re-invite message to incorporate requested information. These alterations will be useful in ensuring availability of user's handset capabilities to IMS core network.
\end{abstract}

Index Terms - quality of service, IP multimedia subsystem, SIP, RTCP, SBC

\section{INTRODUCTION}

Next Generation Network is getting attraction from the service providers. In order to provide multiple services over a single converged network, most of Mobile and fixed network operator had already moved their Telecommunication infrastructure to All IP converged network. IP Multimedia Subsystem (IMS) standardized by 3 GPP is getting attention for replacing the legacy circuit switched network with All IP network [1]. IMS provides Multiple services independent of underlying access network. The user can access the services of IMS using any of access technology which is DSL, Wi-Fi, WiMax, GPRS etc.

IMS is multi service network and user requirement may vary from simple voice call to high multimedia applications. These requirements introduce the significant challenges as network operator must manage real time services across the more complex Multivendor, multiaccess and multi service environment to continuously fulfill the requirement of customers. For providing on demand services to end-user most of the network operator signed SLA agreement with end-user to ensure there required service demands.

In this paper we provide a mechanism for resource reservation by analyzing the traffic stats of end-user. For allocating the resources and QOS services to end-user; we are using Session Border Controller (SBC) that continuously monitored the real-time traffic and RTCP packets for QoS decisions [2]. The SBC also analyzed the SLA of access and core IMS network and enforce polices according to signed SLA and feedback from RTCP packets. As SIP/SDP is core protocol for exchange of signaling information [3] [4] [5]; we are proposing change in invite and re-invite SIP messages for including user specific information's. As IMS services can be accessed from any of access network and SLA's of both access and IMS network are different for this we recommended that the user's access level SLA exchanged with core IMS network and IMS enforce policies accordingly.

The rest of the paper is organized as in section 1 we will discuss the QoS challenges, basic QoS Metrics and provide QoS requirement for different applications. In section 2 we will detail out the functional architecture of IMS and different protocols used in IMS network. In section 3 we will discuss the Policy based architecture for IMS network and State of the art, in section 4. In section 5 we will discuss real-time resource allocation based on session border controller (SBC) with final conclusion retrieved from this study.

\section{BACKGROUND}

In order to highlight the framework for real time resource allocation in IP Multimedia Subsystem Network it is useful to elaborate the preliminary concepts related to these technologies. We have divided this section into four sub sections to explain each of the subsection in detail.

\section{A. QOS CHALLENGES}

IMS is multi-service network that provide data, video, and voice service to end user regardless of under lying access network. The applications are varied from simple 
low quality voice call to high quality multimedia application and require the acceptable service quality from operators. As Multimedia applications are becoming richer day by day and required more bandwidth to fulfill the needs of end-user. In order to meet this; network operator adds more bandwidth to their network but addition of bandwidth does not means that the multimedia application will get the better services from network operators. Besides adding more bandwidth to the network it is also required to provide some strong policing, traffic reshaping, and queuing methods that provides uninterrupted service to end user. In order to provide the good services to customer the aim of Operator is to minimize the jitter, delay, packet loss and maximize the Throughput. The operators ensure the services to end-user within the signed SLA and QOS benchmark. Table 1 provides the QOS bench mark for different applications.

Table 1: QoS Bench Mark (Bandwidth, Delay, Jitter)

\begin{tabular}{|l|l|l|l|}
\hline \multirow{2}{*}{ Applications } & \multicolumn{3}{|c|}{ QoS Benchmark (Sensitive to) } \\
\cline { 2 - 4 } & Bandwidth & Delay & Jitter \\
\hline VoIP & High & Delay & Low \\
\hline VOD & High & Delay & Low \\
\hline IP TV & High & Delay & Low \\
\hline Web & Medium & Medium & Medium \\
\hline Emails & Medium & Medium & Medium \\
\hline IM Messaging & Low & Delay & Low \\
\hline Conferencing & High & Delay & Low \\
\hline PoT & Medium & Delay & Low \\
\hline Database & Moderate & Delay & Medium \\
\hline Banking & Medium & Delay & Low \\
\hline E-Government & Medium & Delay & Low \\
\hline Signaling & Low & Low & Low \\
\hline
\end{tabular}

Table 2: QoS Bench Mark (Packet Loss, Throughput, Availability)

\begin{tabular}{|l|l|l|l|}
\hline \multirow{2}{*}{ Applications } & \multicolumn{2}{|c|}{$\begin{array}{c}\text { QoS Benchmark } \\
\text { (Sensitive to) }\end{array}$} & \multirow{2}{*}{ Availability } \\
\cline { 2 - 3 } & $\begin{array}{c}\text { Packet } \\
\text { Loss }\end{array}$ & Throughput & \\
\hline VoIP & Low & High & High \\
\hline VOD & Low & High & High \\
\hline IP TV & Low & High & High \\
\hline Web & Low & High & Medium \\
\hline Emails & Low & High & Medium \\
\hline $\begin{array}{l}\text { IM } \\
\text { Messaging }\end{array}$ & Low & High & High \\
\hline Conferencing & Low & High & High \\
\hline PoT & Low & High & High \\
\hline Database & Low & High & High \\
\hline Banking & Low & High & High \\
\hline $\begin{array}{l}\text { E- } \\
\text { Government }\end{array}$ & Low & High & High \\
\hline Signaling & Low & High & High \\
\hline
\end{tabular}

\section{B. STATIC VERSUS ONLINE QOS}

Static QoS requires manual setup and reserve the bandwidth as expected by the end user. This will result in degradation when more bandwidth is required as well as wastage of bandwidth when it cannot be used. The main drawback of this approach is that it requires end to end configuration of network to reserve the resources at all network nodes. In contrast, Dynamic QoS does not reserve any bandwidth instead it dynamically processes voice, video, and data traffic on the basis of current needs, optimizing the bandwidth resources.

\section{IP MUltimeida SubSYSTEM}

3GPP defines number of components for IMS network [6], [7], [8]. 3GPP provide the IMS framework for its $3^{\text {rd }}$ generation UMTS network. In conjunction with 3GPP ETSI working group TISPAN [14], [15] provides working architecture for fixed network.

The main functional component of IMS is

Call Session Control Function (CSCF)

Session controller manages SIP session and coordinates with other network entities for session control service control and resource allocation. To fulfill such functionalities, the CSCF can act as Proxy CSCF (P$\mathrm{CSCF}$ ), Serving CSCF (S-CSCF) or Interrogating CSCF (I-CSCF).

- P-CSCF is the first point for contact for end user, end user registers with $\mathrm{P}-\mathrm{CSCF}$ to access the network resources.

- S-CSCF control and maintains services requested by the user. S-CSCF is also responsible for enforcing the policies and finds which application server to be contacted to fulfill the user requirement.

- I-CSCF acts as initial point of contact to the IMS home network from other networks. It routes received SIP requests to the S-CSCF assigned to the user or selects an S-CSCF if one is not currently assigned.

Call Session control functions uses the SIP protocol for exchange of session information between different call session entities as well as with user. SDP is used along with SIP to provide the information about the session and RTP is used for real time traffic.

The Home subscriber server (HSS) provides the authentication, authorization and contains the information about end user and their subscription profiles. HSS use the diameter protocol for authorization, authentication and accounting. Policy Decision Function (PDF) provides QoS policy and resource admission control for the IMS network. PDF uses the services of COPS for exchange of policy information between PEP and PDF.

Other core component of IMS are MGCF that provides interworking with other circuit switched PSTN and PLMN network it controls the Media Gateway that control the user traffic to circuit switched network and signaling gateway that controls signaling exchange with PSTN and PLMN network. MGCF make use of MGCP for signaling to Media gateway and Sigtran for exchange of SS7 Signaling with PSTN. Application server provides services like VOD, presences services, gaming capability, Value added services and IPTV service. MRF provides multiparty conferencing and announcement services. 


\section{POLICY BASED QOS IN IMS}

IMS adopted the Policy based QOS model proposed by IETF RFC [7] to provide the Quality of services for its UMTS based IMS network. The Policy based network is based on two elements, the Policy Decision Point (PDP) and the Policy Enforcement Point (PEP). The role of PEP is to enforce that only authorized IP flows can pass through the network. The PEP send the policy requirement to $\mathrm{PDP}$, the $\mathrm{PDP}$ checked the policy rules for the request and extract a set of policies that has been implemented. The PDP responds back the PEP with the set of rules that has been implemented and PEP enforces the rules that have been extracted from PDP.

The flow of call using policy based network is as under

1. The user send invite message to P-CSCF with SDP information, the S-CSCF routes this to HSS for initial filtration and user authentication and call is routed to the second user.

2. The Second user replies the SIP invite message with SDP information, The P-CSCF examines the SDP information responded by User $\mathrm{B}$ to determine the QoS of service requirement for session. The P-CSCF send request to PCF for token authorization, PDP in IMS serves as a PCF. The PCF replies with token identifying the authorized session and resources. The $\mathrm{P}-\mathrm{CSCF}$ sends this authorization token to caller.

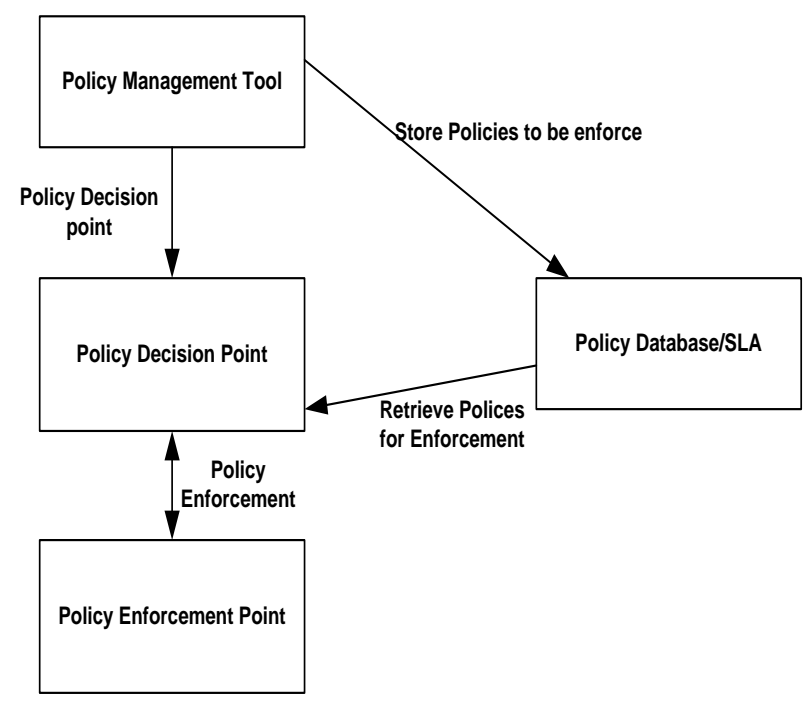

Figure 1.Policy Base Network [8]

3. The caller sends the PDP context activation and token to GSGN and reserve resources

4. PCF check the validity of session after receiving the COPS message of from GGSN.

5. The PCF sends resource reservation information to GGSN. The PEF in the GGSN act as a gate that it controls the flow of packets. It only allows the packets to cross the network that are authorized by the PCF and block the rest of the packets.

The IMS session setup starts with exchange of signaling information to Proxy call session controller, once the signaling setup is completed then media channel is open for the exchange of actual multimedia traffic. The path for signaling and real time traffic is different from each other. As the access to IMS network can be made by using any of the access technology that is Wimax, wifi, GPRS, DSL technologies. Initially 3GPP designed IMS network for UMTS network but later ETSI TISPAN recommends the IMS network for it All IP NGN network.

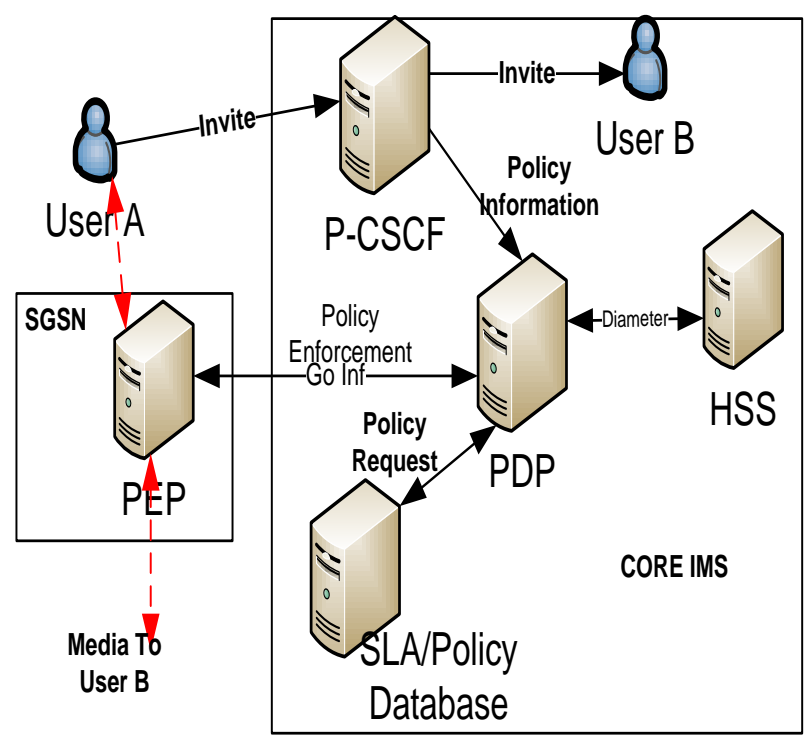

Figure 2. Policy Base Architecture for IMS

\section{RELATED WORK}

As stated earlier 3GPP adopted the IETF policy based QoS for its IMS network. Different proposal has been made to provide the QoS. For UMTS based access to IMS network the [8] proposed the policy based admission control architecture for IMS network. The architecture only take care of Qos requirements for UMTS based end user.3GPP release 6 and release 7 introduce the WLAN access an DSL access to IMS network. [9] Proposed QOS service architecture for WLAN based access to IMS core network. It introduces the implementation of PDP for WLAN network.

There are two segments of network, one is core network and other access network [10]. The proposed packet data gateway is designed on such architecuter to keep both of them separte by means of inclusion of entry points into IMS network. The PDG interfaces with PDF for controlling the resources of network irrespective of access technology.

Managing resources for the end-user during mobility is very difficult task and require much of researcher's attentions. The [11] provides the framework for QOS management for mobile user as well as for all type of access network. The framework introduced the local PDP and PEP nodes in all access networks.

The feedback of quality perceived by user to network also is an important factor for providing better QOS and resource allocation to end user. [12] propose a set-top box architecture that provides the perceived quality of end user to IMS network. The framework did not consider any under lying access network.

The SIP protocol is main signaling protocol for session setup in IMS network. The [13] make use of information 
in SIP messages and proposed almost similar architecture as proposed by. The author proposes to setup local policy decision in access network controlled by border gateways of access network. In addition to functional architecture the author also proposed the changes in SIP messages to incorporate some end user related information i.e., the user access network information, battery timing of mobile, received signal strength from access network etc.

\section{DYNAMIC QOS FRAMEWORK FOR IMS}

Session controller in our architecture is shown in Fig. 4 is B2B user agent. The Main function of session border controller is to provide access of IMS network to end user, monitored traffic and analysis of QOS parameters received by end user. It means Session Border Controller (SBC) provides the two type of functionality. First, analyzed the SLA of access and core IMS network and enforce polices according to signed SLA.

The SBC also act as PEP and negotiate with access network as well as IMS PDP for SLA signed policies. As IMS network can be accessed from different access network and we ensured that the providers of access network agrees to provide SLA of their users. SBC use COPS protocol for getting information about user access SLA.

The second functionality is to continuously monitor the real-time traffic and RTCP feedback packets for QoS decisions. QoS decisions are based on QoS parameters in which to minimize the jitter, delay, packet loss and maximize the throughput.

The SBC continuously monitored the flow of traffic and analyzed the stats received by end user by analyzing the RTCP packet and adjust the resources according to signed SLA.

The Signaling flow for call setup using resource reservation is shown in Fig. 4.

1. Initially user sends invite message to P-CSCF with SDP information. Session border controller relays the request to $\mathrm{P}-\mathrm{CSCF}$ for initial filtering.

2. P-CSCF sends invite message to S-CSCF. The $\mathrm{S}$-CSCF routes this to HSS for initial filtration and user authentication.

3. After filtration and authentication, S-CSCF sends the SIP invite message to the second user.

4. The Second user replies the SIP invite message with SDP information.

5. The P-CSCF examines the SDP information responded by User B to determine the QoS of service requirement for session.

6. The P-CSCF responded back with SDP information of the called user. a. The P-CSCF send request to PCF for token authorization, PDP in IMS serves as a PCF.

b. PCF replies with token identifying the authorized session and resources.

c. The P-CSCF sends this authorization token to caller.

7. The SBC enquires the PDP for policies which the PDP response back and the SBC with required resources for Session.

a. The PEP uses the COPS protocol for negotiating policies from access network and IMS PDP.

- The caller sends the PDP context activation and token to GSGN and reserve resources.

- $\quad$ PCF check the validity of session after receiving the COPS message of from GGSN.

- The PCF sends resource reservation information to GGSN.

- The PEF in the GGSN act as a gate that it controls the flow of packets. It only allows the packets to cross the network that are authorized by the PCF and block the rest of the packets.

8. After reservation of resources for session the two media session was open between the end users.

9. After the session establishment, if the end user requirements are changed during the transmission especially in mobility cases then end user sends the SIP re-invite message which contains the user new required data.

The SBC relays the real time traffic between the end user continuously monitor the RTCP packets for getting the perceived quality of flows and QOS stats. The SBC drops the packets that are not according the policies provided by the PDP. If the resources for particular session need to be changed the SBC directly request the PDP for resource reservation and PDP respond back result after negotiating the HSS and Policy server.

The end-user capabilities help IMS network efficient allocation of resources. Mani et al., [13] proposed an alteration in SIP messages to incorporate the user related information during resource allocation. We suggest inclusion of user access network information in SIP invite message. During the conversation and mobility the network resources are changed then the new resource allocations are required so, the IP re-invite message is sent containing user information about access network and battery timing. 


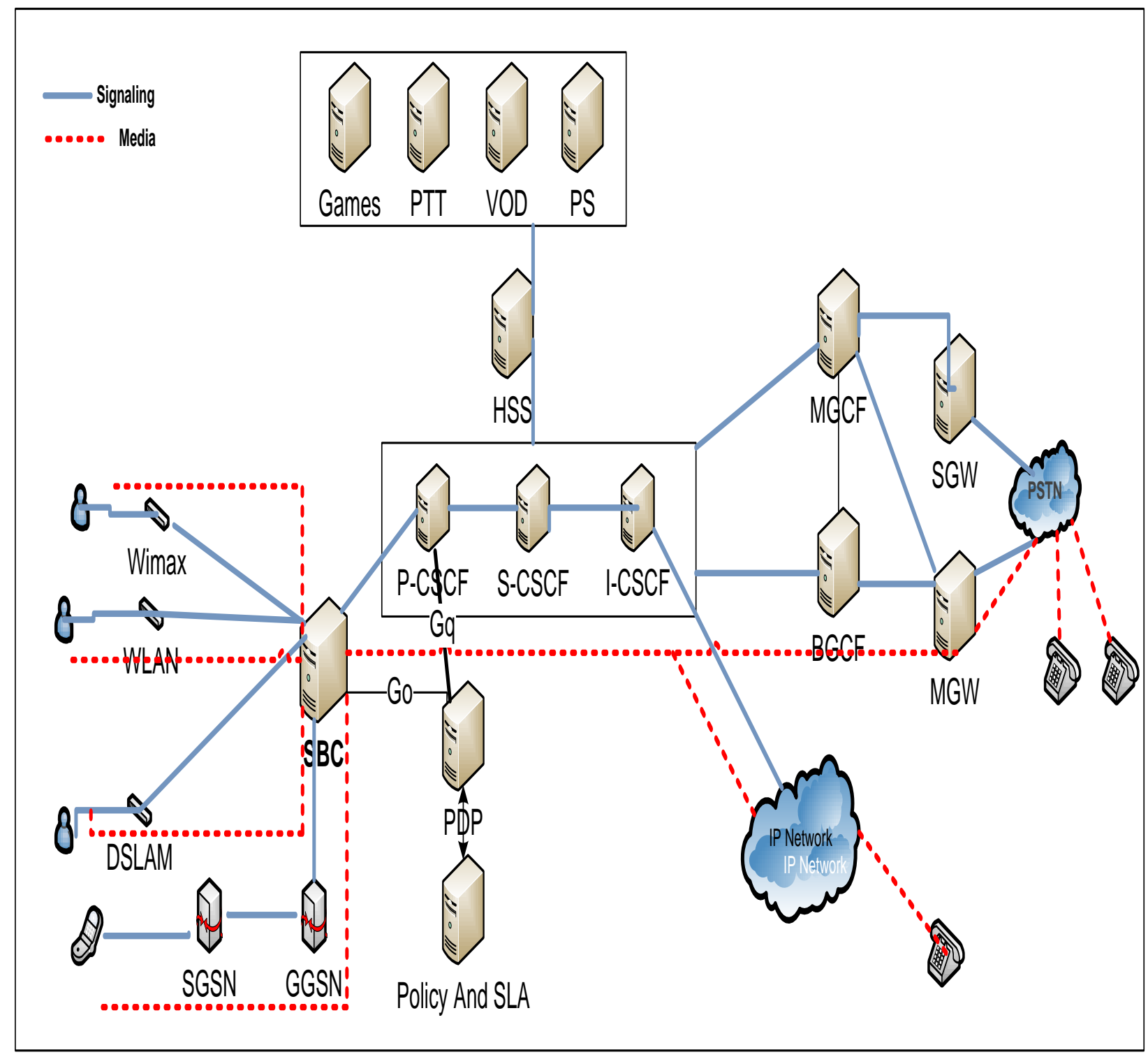

Figure 3. Real Time Resource Allocations 


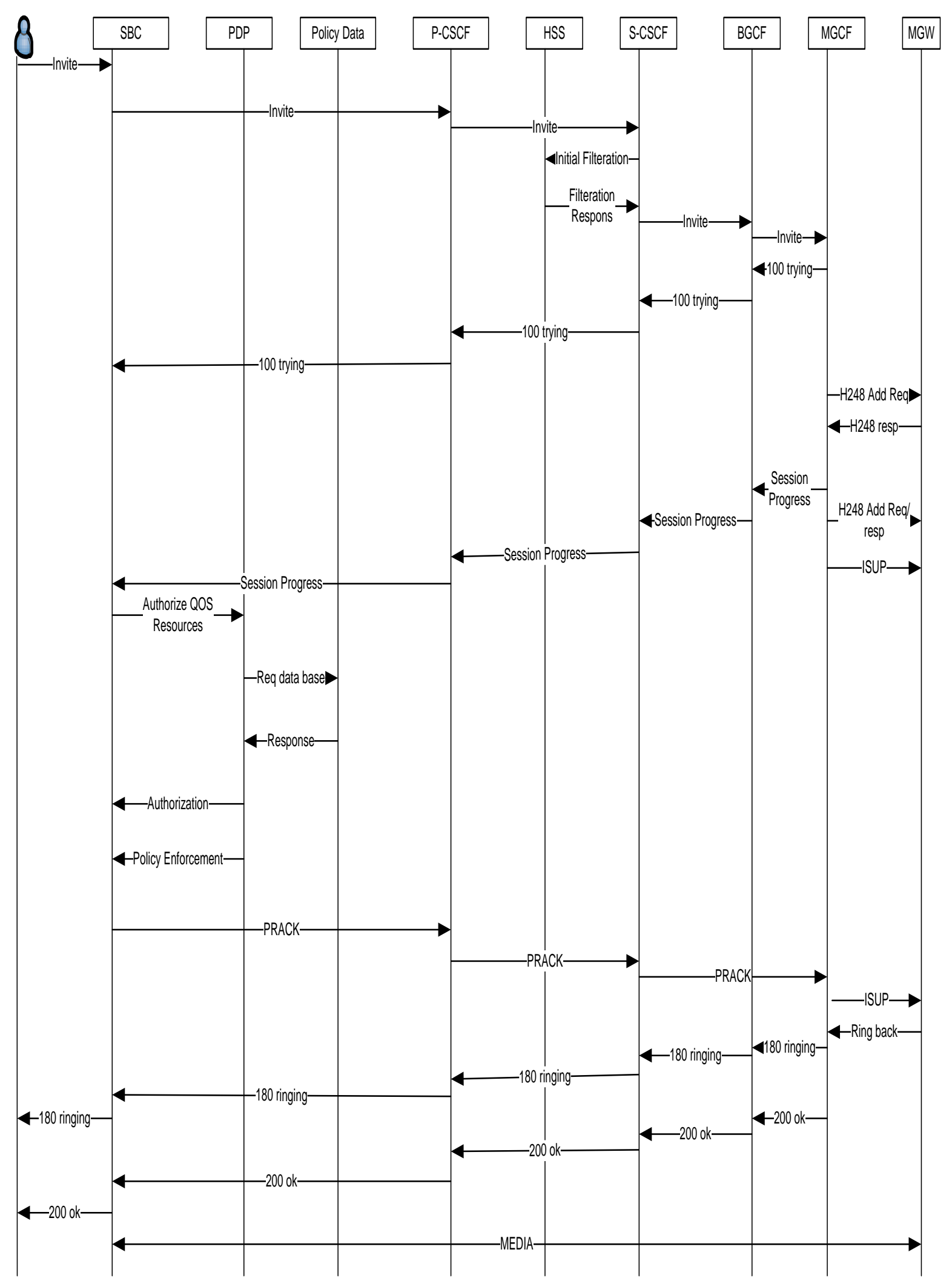

Figure 4. Call Flow for Resource Allocation Conclusion

\section{CONCLUSIONS}

In this study, we proposed a architecture which based on Session border controller (SBC) that interact with access network operator for getting user's SLA and reserve resources accordingly. We also introduced a real time resource allocation mechanism depending upon the network RTCP feedback report. 
SBC continuously monitored the RTCP packet and allocate resources to end user in conjunction with information received from access network and IMS SLA. We also proposed an alteration in SIP invite and re-invite message to include user specific information during resource allocation process. Moreover, we have also shown that how this framework is useful for Real-Time Resource Allocation in IP Multimedia Subsystem Network

\section{REFERENCES}

[1] 3rd Generation Partnership Project, TS 23.228: Release 7, IP Multimedia .Subsystem (IMS) (http://www.3gpp.org).

[2] Schulzrinne H., Casner S. RTP Profile for Audio and Video Conferences with Minimal Control. Request for comments 3551, Internet Engineering Task Force, 2003

[3] Rosenberg, J., et al., SIP:Session Initiation Protocol, IETF RFC 3261, June 2002

[4] Rosenberg, J. and H. Schulzrinne, An Offer/Answer Model with Session Description Protocol (SDP)“", IETF RFC 3264, June 2002

[5] Magedanz, T., and De Gouveia, F. C. (2006). IMSthe IP multimedia system as NGN service delivery platform. e \& i Elektrotechnik und Informationstechnik, 123(7), 271-276.

[6] Knightson, K., Morita, N., \& Towle, T. (2005). NGN architecture: generic principles, functional architecture, and implementation. Communications Magazine, IEEE, 43(10), 49-56.

[7] IETF RFC 2753, "A Framework for Policy-Based Admission Control," 2000.

[8] Zhuang, W., S. G. Yung, K. J. Loh, and K. C. Chua., Policy-based QoS architecture in the IP multimedia subsystem of UMTS.IEEE Network, 17(3):51-57, May/June 2003

[9] Zhuang, W., Y. Gan, K. Loh, and K. Chua, "Policybased QoS management Architecture in an integrated UMTS and WLAN environment," IEEE Communications Magazine, pp. 118-125, November 2003

[10] An End to End QoS Management Framework for the 3GPP IP Multimedia Subsystem R. Good, N. Ventura Proceedings of the 2007 IEEE International Conference on Telecommunications and Malaysia International Conference on Communications, 14-17 May 2007, Penang, Malaysia

[11]De Gouveia, F. C., \& Magedanz, T. (2005, December). A framework to improve QoS and mobility management for multimedia applications in the IMS. In Multimedia, Seventh IEEE International Symposium on (pp. 7-pp). IEEE.

[12] Siddiqui, M. S., Amin, S. O., \& Hong, C. S. (2009). A set-top box for end-to-end QoS management and home network gateway in IMS. Consumer Electronics, IEEE Transactions on, 55(2), 527-534.

[13] Mani, M., and N. Crespi. New QoS control mechanism based on extension to SIP for access to UMTS core network via different kinds of access networks. In Wireless and Mobile Computing, Networking and Communications, 2005. (WiMob'2005), IEEE International Conference on, volume 2, pages 150-157, August 2005

[14] Blum, N., Boldea, I., Magedanz, T., \& Margaria, T. (2010). Service-oriented Access to Next Generation Networks-from Service Creation to Execution. Mobile networks and applications, 15(3), 356-365.

[15] Cruz, R. A. S., Nunes, M. S., Menezes, L., \& Domingues, J. (2011). IPTV architecture for an IMS environment with dynamic QoS adaptation. Multimedia Tools and Applications, 53(3), 557-589.

Muhammad Naeem: Research scholar at department of computer science, M. A. Jinnah University Islamabad Pakistan. His research area includes computer networks, machine learning, semantic computing, text retrieval and data mining.

Shahneela Naz: Research scholar at department of computer science, M. A. Jinnah University Islamabad Pakistan. Her research area includes Clustering Classification, Semantic engineering, Text retrieval, Adaptive Real-time Video Multicast Techniques, Layered Video Multicast, Multimedia protocols, Routing and Multimedia Streaming.

Saira Gilani: She received her M.IT degree in Information Technology from Balochistan University, Quetta, Pakistan in 2004. She is an MS student at M.A. Jinnah University, Islamabad, Pakistan and is a member of Center of Research in Networks and Telecommunication (CoReNeT). Her current research activities include investigation of MAC layer schemes, network security for Vehicular Ad Hoc Networks and data mining and semantic computing. 